\title{
USO DE LAS TIC EN EL PROCESO DE ENSEÑANZA PARA LOGRAR EL APRENDIZAJE SIGNIFICATIVO EN LOS ALUMNOS DE INSTITUCIONES EDUCATIVAS PERUANAS DEL NIVEL SECUNDARIO
}

Mg. Jenny V. Moreno Sáenz Docente de Estudios Generales

Mg. Liliana Espinoza Herrera Colaboradora

\section{Introducción}

El trabajo que se presenta en este artículo se desarrolla en el marco de la Norma Internacional ISO 9004: 2009: que es una herramienta de autoevaluación para mejorar la calidad de las organizaciones y la propuesta de Andrew Churches: La taxonomía digital, basada en la Taxonomía de Bloom. Ambos enfoques, aplicados adecuadamente permiten hacer uso eficiente de las TIC para lograr el aprendizaje significativo en los alumnos de educación básica regular del nivel secundario.

La Norma Internacional ISO 9004: 2009 presenta un marco general, para el desarrollo de este trabajo se ha tomado en cuenta solo algunos criterios que han sido contextualizados para el uso de las TIC en el proceso de enseñanza, considerando el actual modelo educativo del nuevo diseño curricular. Asimismo los criterios de la norma se ha articulado con la Taxonomía de Bloom para la era digital, que al ser aplicados se puede lograr el aprendizaje significativo del alumno.

\section{El uso de las TIC}

El uso de las TIC (Tecnologías de la Información y Comunicación) se ha convertido actualmente en uno de los pilares básicos de la sociedad porque promueve la integración del conocimiento y por la gran influencia que ejerce sobre el ámbito educativo, es por ello, necesario proporcionar a los estudiantes una educación que tenga en cuenta esta realidad, 
Para tener una educación acorde a un mundo globalizado en donde las TIC tienen un rol preponderante es importante, sobre todo que los maestros sepan utilizarla en los procesos de enseñanza aprendizaje; para ello deben de conocer la herramienta tecnológica, saber manejarla y saber cómo usarla en una sesión de clase para lograr el aprendizaje significativo en sus estudiantes.

\section{El aprendizaje significativo}

Es aquel aprendizaje que queda en el alumno de manera permanente, es un proceso mental de adquirir contenidos conceptuales, interiorizarlos y posteriormente generar conocimientos.

El alumno al captar, conocer, comprender, decidir y actuar se convierte en el principal protagonista y responsable de su propio aprendizaje, construyendo sus conocimientos y elaborando aprendizajes nuevos desde sus experiencias previas.

Los aprendizajes al ser interiorizados por el alumno y asumirlo como importante para su vida, permite el cambio permanente en el desarrollo de sus capacidades y habilidades para lograr ser competente.

Según Vigotsky, los aprendizajes no son sólo procesos intrapersonales, sino fundamentalmente interpersonales.'

Según Novak, el aprendizaje es tomar conciencia de lo que desea aprender y cómo es que aprende (metacognición). Esto le permite al alumno a descubrir sus potencialidades y limitaciones y le posibilita ser capaz de enfrentar las dificultades que se le presente con mayor éxito, es decir que los alumnos aprendan a aprender. ${ }^{2}$

Precisamente muchas de las herramientas educativas tecnológicas, se han basado en la metodología de aprender haciendo, que tiene un enfoque netamente constructivista, porque es el aprendizaje que se descubre por sí mismo a través de la vivencia e interactuando con su entorno.

\section{Condiciones para producirse el aprendizaje significativo utilizando las TIC}

Para lograr el aprendizaje significativo, se debe de contar con ciertos requisitos a cumplirse como:

- Lo que se pretende enseñar, el alumno debe de percibirlo útil, ameno y debe de estar relacionado con sus saberes previos, de tal manera que desee indagar más en el tema.

1 Vygotsky, L. S. (1982). Historia del desarrollo de las funciones psíquicas superiores. Ed. Pueblo y Educación, La Habana.

2 Novak, P. \& Hanesian, H. (1989). Psicología educativa. Un punto de vista cognitivo. México : Trillas. 
- Para desarrollar el proceso pedagógico se debe de utilizar una herramienta tecnológica adecuada al proceso mismo, esto ayuda a captar la atención de los alumnos.

- En el desarrollo del inicio de una sesión de clase, la problematización debe de tener sentido lógico y usar material tecnológico que sea significativo y organizado para el alumno.

- Disposición por parte de los alumnos a aprender.

- El material tecnológico a usar debe de ser relacionado con la estructura cognitiva del alumno para generar ideas nuevas.

- Las actividades realizadas con las herramientas tecnológicas para desarrollar las capacidades específicas, deben de estar relacionadas con los procesos cognitivos y tener significado lógico.

\section{Taxonomía de Bloom}

La Taxonomía de Bloom consiste en clasificar los objetivos educativos y los ha clasificado en 3 dominios:

Dominio cognitivo: Tiene 6 niveles: conocimiento, comprensión, aplicación, análisis, síntesis y evaluación.

Dominio afectivo: Tiene 5 niveles: recepción, respuesta, valoración, organización y caracterización.

Dominio psicomotor: Tiene 6 niveles: se establecen los siguientes niveles: percepción, disposición, mecanismo, respuesta compleja, adaptación y creación

Andrew Churches actualizó la Taxonomía de Bloom acorde a la era digital ${ }^{3}$, complementando cada categoría con capacidades y herramientas del mundo digital que permitan el desarrollo de habilidades para Recordar, Comprender, Aplicar, Analizar, Evaluar y Crear. Esta propuesta digital de Bloom, se basa en el Dominio Cognitivo, en donde los alumnos para lograr las capacidades debe de poseer habilidades inferiores por ejemplo, para que un alumno sea capaz de aplicar. Ver Figura 1.

3 Churches, A. (2008). Taxonomía de Bloom para la era digital [en línea]. EDUTEKA. Recuperado de: http://eduteka.icesi.edu.co/articulos/ TaxonomiaBloomDigital 

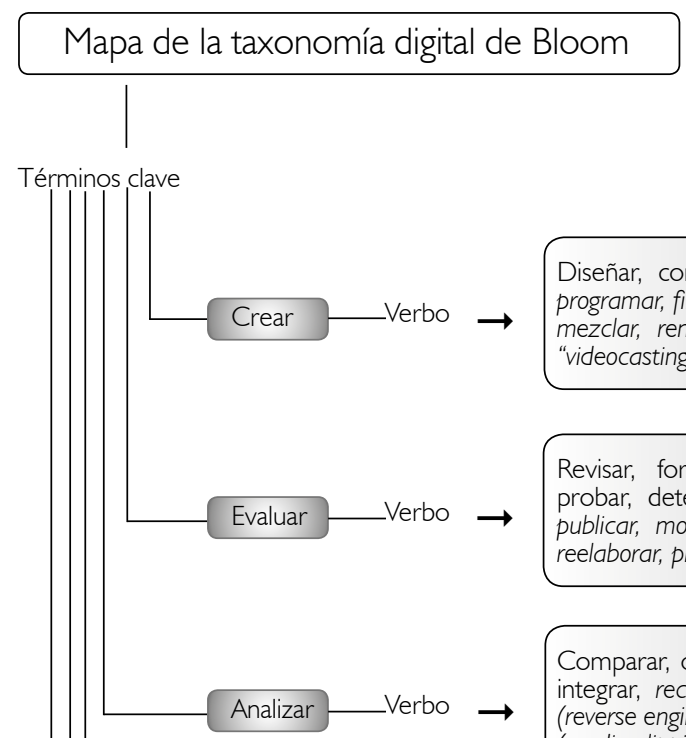

Diseñar, construir, planear, producir, idear, trazar, elaborar, programar, firmar, animar, blogear, video blogear (video blogging), mezclar, remezclar, participar en un wiki (wiki-ing), publicar, "videocasting", "podcasting", dirigir, transmitir.

Revisar, formular hipótesis, criticar, experimentar, juzgar, probar, detectar, monitorear, comentar en un blog, revisar, publicar, moderar, colaborar, participar en redes (networking, reelaborar, probar.

Comparar, organizar, deconstruir, atribuir, delinear, encontrar, integrar, recombinar, enlazar, validar, hacer ingeniería inversa (reverse engineering). "cracking", recopilar información de medios (media clipping).

Implementar, desempeñar, usar, ejecutar, correr, cargar, jugar, operar, "hackear" (hacking), subir archivos a un servidor, compartir, editar.

Interpretar, resumir, inferir, parafrasear, clasificar, comparar explicar, ejemplificar, hacer búsquedas avanzadas, hacer búsquedas Booleanas, hacer periodismo en formato de blog (blog jurnalism), "Twittering" (usar Twitter), categorizar etiquetar, comentar, anotar, suscribir.

Reconocer, listar, describir, identificar, recuperar, denominar, localizar, encontrar, utilizar viñetas (bullet pointing), resaltar, marcar (bookmarking), participar en la red social (social bookmarking), marcar sitios favoritos (favouriting/locla bookmarking), buscar, hacer búsquedas en Google (googling).

\begin{tabular}{|llll|}
\hline \multicolumn{2}{c}{ ESPECTRO DE LA COMUNICACIÓN } \\
Moderar & Reunirse en la red & Publicar y blogear & Comunicarse por Twitter/ \\
Colaborar & Realizar videoconferencias & Participar en redes & Microblogs \\
Moderar & por Skype & Contribuir & Mensajería \\
Negociar & Revisar & Chatear & Instantánea \\
Debatir & Preguntar/cuestionar & Comunicarse por correo & Escribir Textos \\
Comentar & Contestar & electrónico & \\
\end{tabular}




\section{Descripción de la herramienta de autoevaluación ISO 9004 - 2009}

La norma ISO 9004 - 2009 incluye dos tablas de autoevaluación, las cuales son: a) De los elementos claves, y b) Para los elementos detallados. Ambas tablas utilizan cinco niveles de madurez (del I al 5) que son aplicados a las áreas que guardan relación directa con el uso de las TIC. Tal y como se observan en las Tablas I y 2 respectivamente.

La Tabla I de Autoevaluación para los elementos claves, permite evaluar los criterios de la tabla para medir el desempeño actual de la institución y determinar el nivel de madurez en que se encuentra. ${ }^{4}$

Los criterios que se evalúan de los elementos claves son: la Gestión, el Liderazgo, la Estrategia y Políica, los Recursos, los Procesos, los Resultados, el Seguimiento y Medición, la Mejora, innovación y aprendizaje.

La Tabla 2 de Autoevaluación para los elementos detallados, evalúa el desempeño actual de los elementos claves de la institución, pero lo hace de manera detallada, utilizando una tabla para cada elemento clave. I) Gestión para el éxito sostenido, 2) Gestión de los recursos tecnológicos, 4) Gestión de los procesos pedagógicos, 5) Seguimiento, medición, Análisis y revisión de los resultados del aprendizaje, 6) Procesos de mejora, innovación y aprendizaje con prioridad.

En cada tabla se especifican los criterios a evaluar, y para cada criterio se establecen los indicadores para cada uno de los cinco niveles de madurez.

\begin{tabular}{|c|c|c|c|c|c|}
\hline \multirow{2}{*}{ Elementos claves: Criterios } & \multicolumn{5}{|c|}{ Niveles de madurez } \\
\hline & I & 2 & 3 & 4 & 5 \\
\hline ¿Cuál es el centro de interés de la dirección? (Liderazgo) & & & & & \\
\hline ¿Cuál es el enfoque del liderazgo? (Gestión) & & & & & \\
\hline ¿Cómo decidimos qué es importante? (Estrategia y política) & & & & & \\
\hline ¿Qué necesitamos para obtener resultados? (Recursos) & & & & & \\
\hline ¿Cómo se organizan las actividades? (Procesos) & & & & & \\
\hline ¿Cómo se logran los resultados? (Seguimiento y medición) & & & & & \\
\hline ¿Cómo se realiza el seguimiento de los resultados? (Seguimiento y medición) & & & & & \\
\hline $\begin{array}{l}\text { ¿Cómo se deciden las prioridades de mejora? (Mejora, Innovación y } \\
\text { aprendizaje) }\end{array}$ & & & & & \\
\hline ¿Cómo tiene lugar el aprendizaje? (Mejora, Innovación y aprendizaje) & & & & & \\
\hline
\end{tabular}


Tabla I. Autoevaluación para los elementos Claves. ISO 9004-2009

\begin{tabular}{|c|c|c|c|c|c|c|}
\hline \multirow[t]{2}{*}{ Elementos detallados } & \multirow[t]{2}{*}{ Criterios } & \multicolumn{5}{|c|}{ Niveles de madurez } \\
\hline & & 1 & 2 & 3 & 4 & 5 \\
\hline \multirow{4}{*}{$\begin{array}{l}\text { Gestión para el éxito sostenido } \\
\text { de una institución educativa }\end{array}$} & $\begin{array}{l}\text { Gestión para el éxito sostenido. } \\
\text { Generalidades }\end{array}$ & & & & & \\
\hline & Éxito Sostenido & & & & & \\
\hline & Entorno de la Institución & & & & & \\
\hline & $\begin{array}{l}\text { Partes Interesadas, necesidades y } \\
\text { expectativas }\end{array}$ & & & & & \\
\hline \multirow{4}{*}{ Estrategia y política } & Estrategia y política. Generalidades & & & & & \\
\hline & Formulación de la estrategia y política. & & & & & \\
\hline & Despliegue de la estrategia y política. & & & & & \\
\hline & Comunicación de la estrategia y política. & & & & & \\
\hline \multirow{2}{*}{ Gestión de los recursos } & Gestión de los recursos. Generalidades & & & & & \\
\hline & Personal de la Institución Educativa & & & & & \\
\hline Gestión de los procesos & $\begin{array}{l}\text { Planificación y control de los procesos. } \\
\text { Generalidades }\end{array}$ & & & & & \\
\hline \multirow{2}{*}{$\begin{array}{l}\text { Seguimiento, medición, análisis } \\
\text { y revisión }\end{array}$} & $\begin{array}{l}\text { Seguimiento, Medición, Análisis y } \\
\text { Revisión. Generalidades }\end{array}$ & & & & & \\
\hline & $\begin{array}{l}\text { Medición con respecto a los indicadores } \\
\text { claves de desempeño }\end{array}$ & & & & & \\
\hline \multirow{4}{*}{$\begin{array}{l}\text { Mejora innovación y } \\
\text { aprendizaje }\end{array}$} & Innovación y Aprendizaje. Generalidades & & & & & \\
\hline & Mejora & & & & & \\
\hline & Innovación & & & & & \\
\hline & Aprendizaje & & & & & \\
\hline
\end{tabular}

\section{Herramienta de autoevaluación contextualizada para el uso de las TIC aplicando la taxonomía de Bloom para la era digital}

La herramienta de autoevaluación contextualizada se basa en la Norma ISO 9004 2009 que ha sido adecuada con el uso de las TIC de acuerdo al modelo educativo basado en competencias y articulada con la Taxonomía de Bloom para la era digital.

Para adecuar la herramienta de autoevaluación con el uso de las tics y la taxonomía de Bloom para la era digital se han considerado los siguientes insumos 


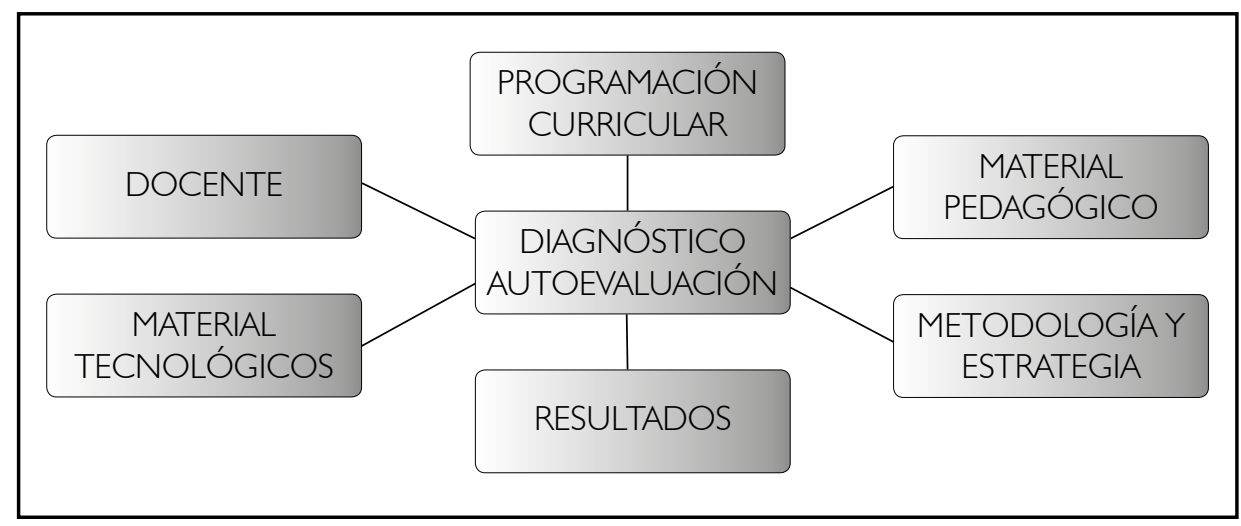

La tabla 3 para los elementos claves se ha considerado los siguientes criterios: Procesos, Estrategia y política y Recursos

La Tabla 4, 5 y 6 de Autoevaluación para los elementos detallados, evalúa el desempeño actual de los elementos con respecto al uso de las TIC, en una sesión de clase, pero lo hace de manera detallada, utilizando una tabla para cada elemento clave,. Para este caso se ha articulado con la Taxonomía I de Bloom para la Era Digital.

En la tesis [Moreno, 20 I 4], se especifican detalladamente las 6 tablas de autoevaluación de elementos detallados, $y$ todos los criterios que intervienen pero sin estar articulado con la Taxonomía de Bloom. 


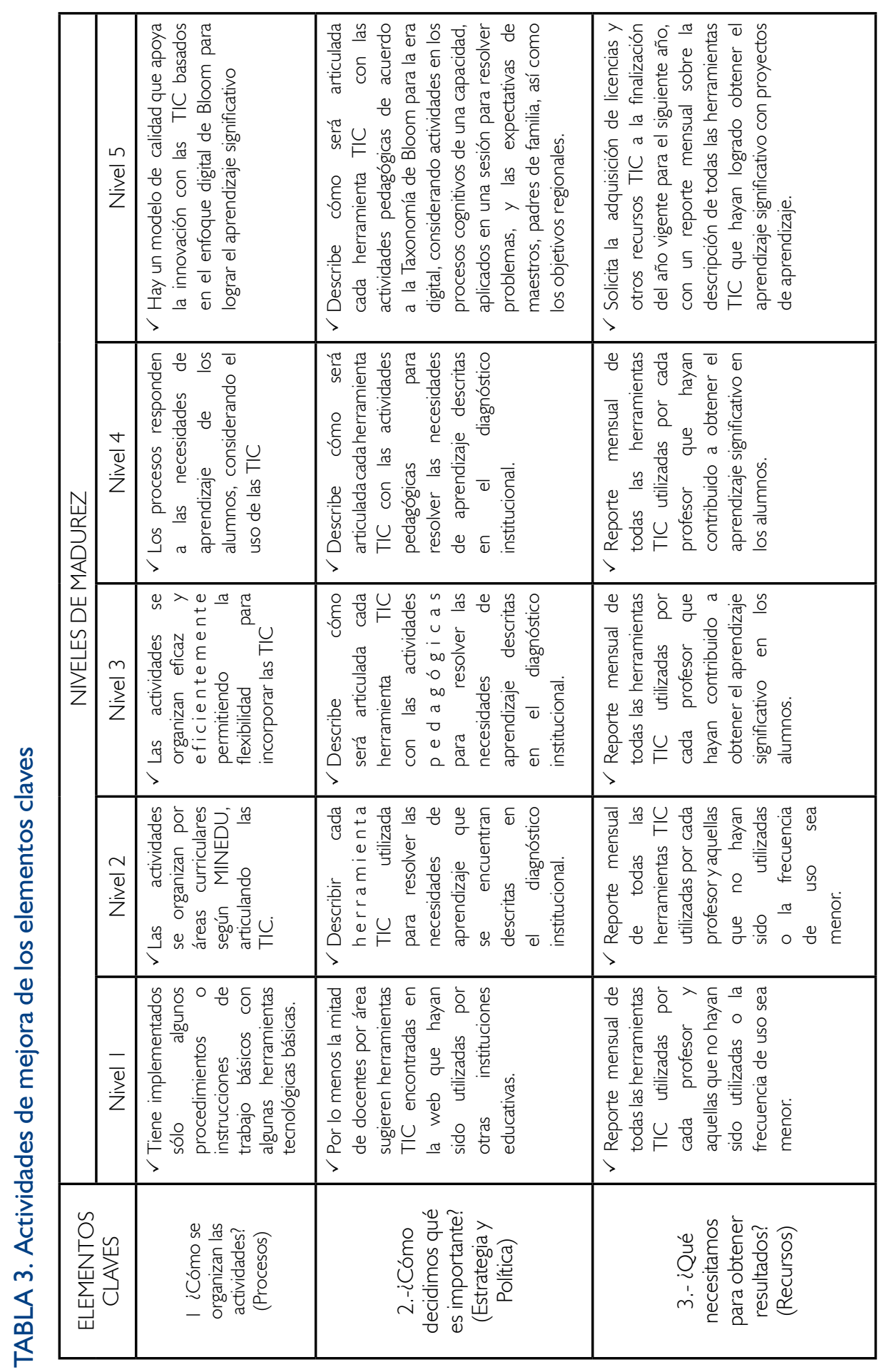




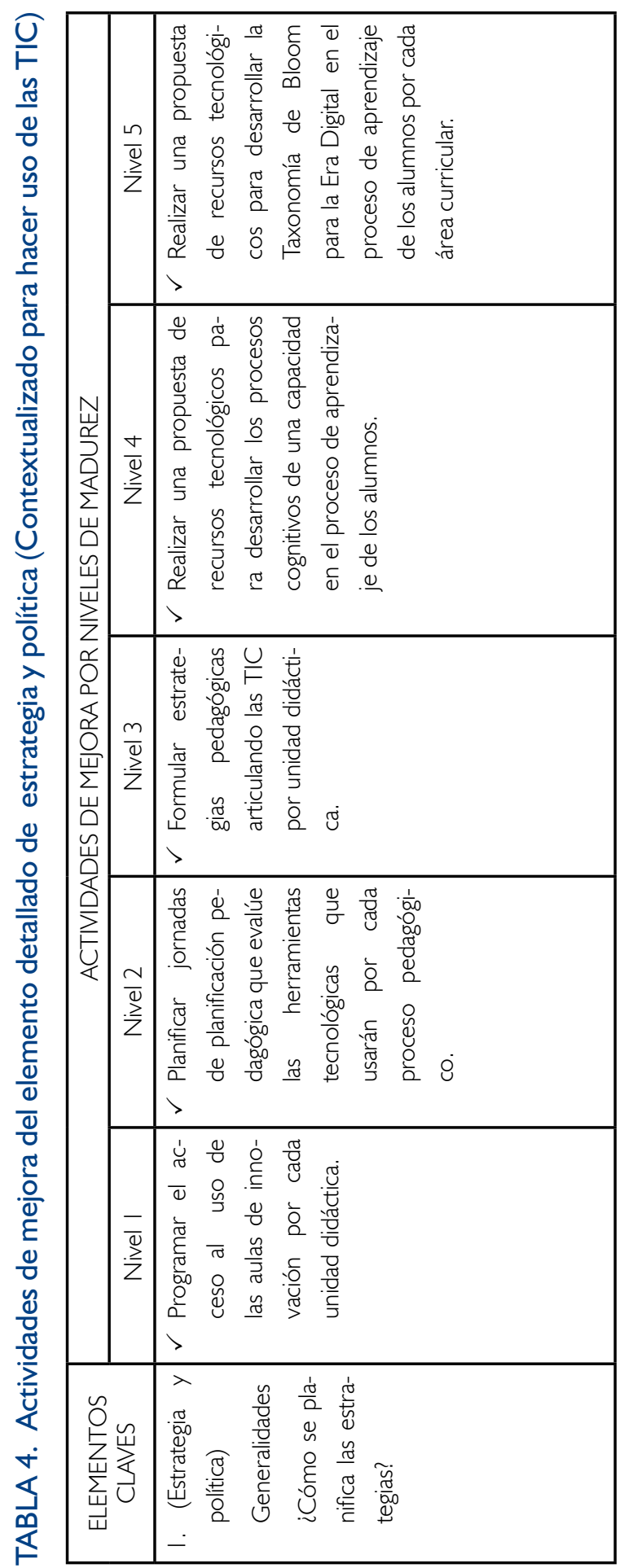




\begin{tabular}{|c|c|c|c|}
\hline \multirow{5}{*}{ 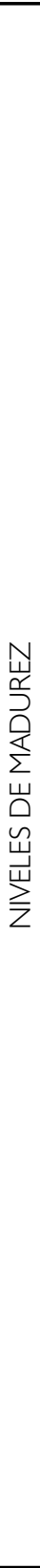 } & $\frac{\stackrel{\sim}{n}}{\stackrel{D}{z}}$ & 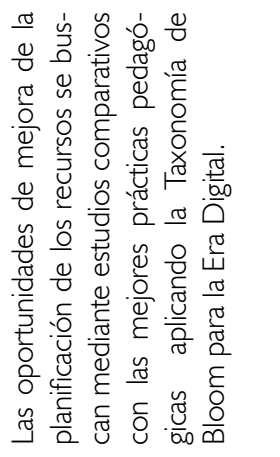 & 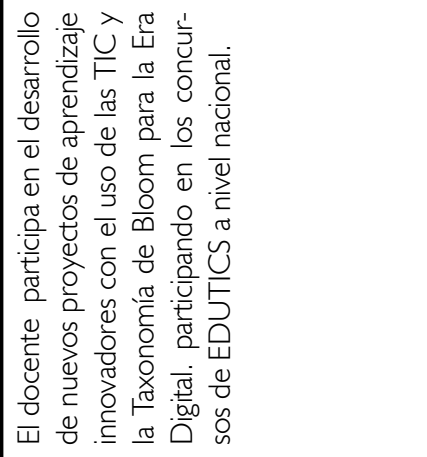 \\
\hline & & 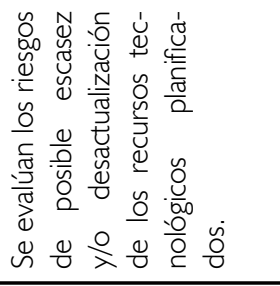 & 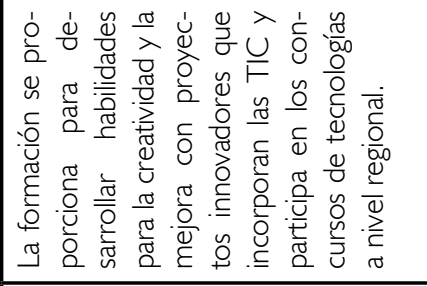 \\
\hline & $\frac{\frac{m}{\nu}}{\frac{D}{Z}}$ & 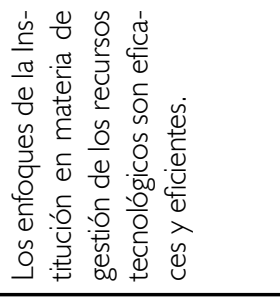 & 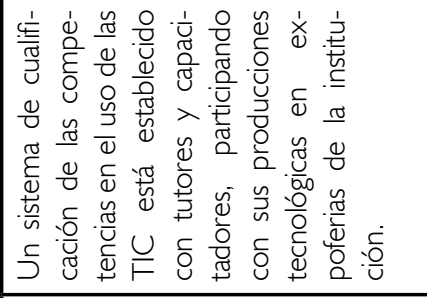 \\
\hline & & 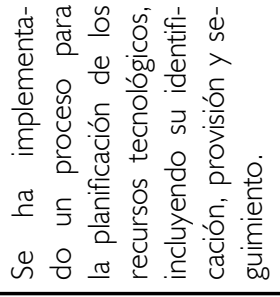 & 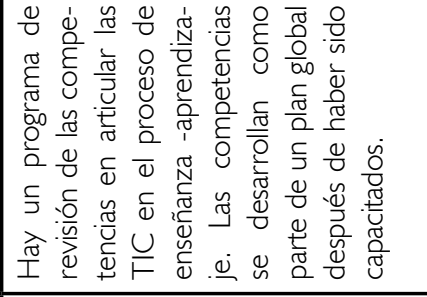 \\
\hline & $\frac{\overline{\bar{D}}}{\overline{\bar{Z}}}$ & 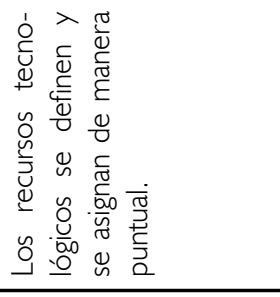 & 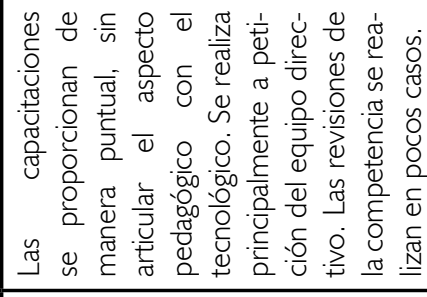 \\
\hline 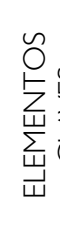 & & 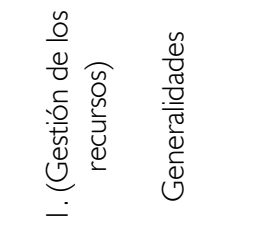 & 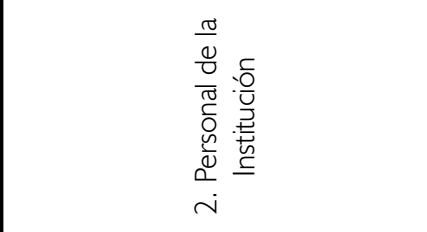 \\
\hline
\end{tabular}




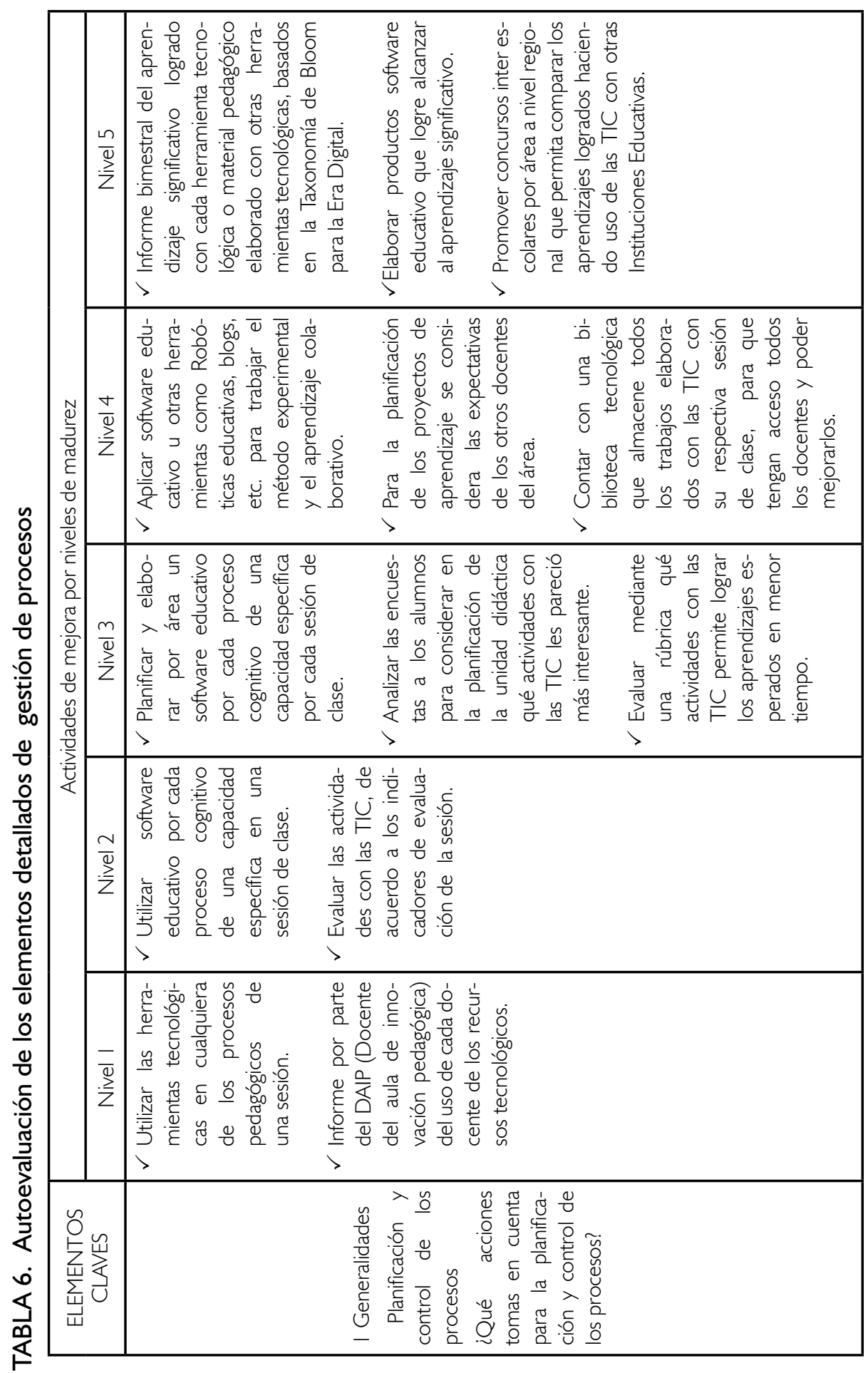




\section{Referencias}

Cabero, J. (2008). Creación de una Guía de Evaluación - Autoevaluación de Centros de Recursos Universitarios de Producción de TIC en la Enseñanza, Revista de medios y educación, N. ${ }^{\circ} 32$.

ISO. Norma Internacional ISO 9004 -2009. (2009). Gestión para el Éxito Sostenido de una Organización. Suiza.

McDonnell, L, De Nieves, C. (2006) Comparación entre los Modelos de Gestión de Calidad Total. X Congreso de Ingeniería de Organización. Valencia.

Moreno, J. (2014) Modelo de Calidad para la Gestión de las TIC en el Proceso de Enseñanza para las Instituciones Educativas Públicas de Educación Secundaria. Facultad de Ingeniería de Sistemas. UNMSM. Lima, Perú.

Churches, A. (2008). Taxonomía de Bloom para la era digital [en línea]. EDUTEKA. Recuperado de: http://eduteka.icesi.edu.co/articulos/TaxonomiaBloomDigital

| 4- | |-20 |6, en: http://eduteka.icesi.edu.co/articulos/TaxonomiaBloomDigital

Ausubel, D. Novak, P., \& Hanesian, H. (1989). Psicología educativa. Un punto de vista cognitivo. México: Trillas.

Vygotsky, L. S. (1 982). Historia del desarrollo de las funciones psíquicas superiores. Ed. Pueblo y Educación, La Habana.

UNESCO (20I3). Enfoques Estratégicos sobre las TIC en Educación en América Latina y el Caribe. 